\title{
A REVIEW ON FTP CLIENT/SERVER TECHNOLOGY
}

\section{Vipin Kumar ${ }^{1 *}$ and Tarun Dalal ${ }^{2}$}

${ }^{* 1} M$. Tech Scholar, CBS Group of Institutions, CSE Department, MDU Rohtak, India

${ }^{2}$ Assistant Professor, CBS Group of Institutions, CSE Department, MDU Rohtak, India

*1Email: er.vipinsharma13@gmail.com, ${ }^{2}$ Tarundalal88@gmail.com

*Corresponding Author: -

Email ID - er.vipinsharma13@gmail.com

\begin{abstract}
: -
FTP is built on a client-server architecture and uses separate control and data connections between the client and the server. FTP users may authenticate themselves using a clear-text sign-in protocol, normally in the form of a username and password, but can connect anonymously if the server is configured to allow it. For secure transmission that hides (encrypts) the username and password, and encrypts the content, FTP is often secured with SSL/TLS ("FTPS"). SSH File Transfer Protocol ("SFTP") is sometimes also used instead, but is technologically different.
\end{abstract}

Keywords: - Review, ftp, client/server, technology

\section{(c) $\$(1)$}




\section{INTRODUCTION TO FTP}

FILE TRANSFER PROTOCOL allows you transfer files between two computers on the Internet. FTP is a simple network protocol based on Internet Protocol and also a term used when referring to the process of copying files when using FTP technology.

\section{FTP CLIENT / SERVER}

To transfer files with FTP, you use a program often called the "client." The FTP client program initiates a connection to a remote computer running FTP "server" software. After the connection is established, the client can choose to send and/or receive copies of files, singly or in groups. To connect to an FTP server, a client requires a username and password as set by the administrator of the server. Many public FTP archives follow a special convention for that accepts a username of "anonymous." FTP allows you to transfer files between two computers on the Internet. FTP is a simple network protocol based on Internet Protocol and also a term used when referring to the process of copying files when using FTP technology.

The first FTP client applications were command-line applications developed before operating systems had graphical user interfaces, and are still shipped with most Windows, Unix, and Linux operating systems. Dozens of FTP clients and automation utilities have since been developed for desktops, servers, mobile devices, and hardware, and FTP has been incorporated into hundreds of productivity applications, such as Web page editors.

\section{MODES OF TRANSMISSION}

FTP supports two modes of data transfer:

- Plain text (ASCII)

- Binary

A common error when using FTP is attempting to transfer a binary file (such as a program or music file) while in text mode, causing the transferred file to be unusable.

\section{FTP SERVER}

An FTP server is a required software application on networks that wish to support file sharing using the File Transfer Protocol.

\begin{tabular}{lcl}
\hline \multicolumn{1}{c}{ Name } & \multicolumn{1}{c}{ Platform } & \multicolumn{1}{c}{ Details } \\
\hline ALFTP & Windows only & $\begin{array}{l}\text { Personal FTP } \\
\text { server }\end{array}$ \\
$\begin{array}{l}\text { Cerberus } \\
\text { FTPS, }\end{array}$ & Windows only & \multicolumn{1}{c}{ FTP, } \\
$\begin{array}{l}\text { FTP SFTP, } \\
\text { Server }\end{array}$ & HTTPS web & \\
& & client, IPv6, \\
& & SOAP-based \\
& & web services \\
& & API, \\
& & Windows \\
& & Active \\
& & Directory / \\
& & LDAP
\end{tabular}




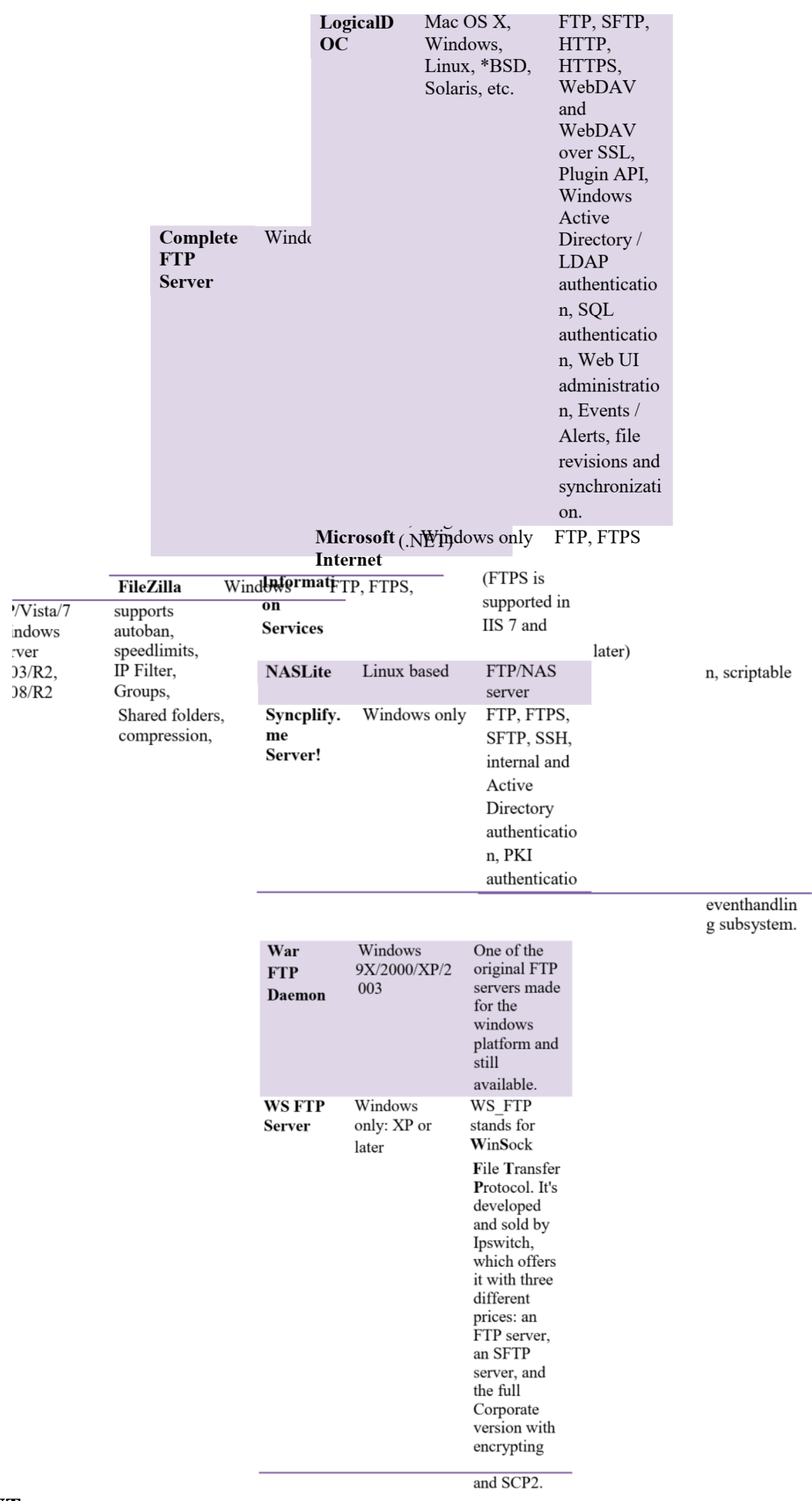

\section{FTP CLIENT}

An FTP client supports downloading of files across the Internet from computers called FTP servers. FTP stands for File Transfer Protocol, the technology that allows FTP clients and servers to communicate over computer networks. An FTP client is a software program with a GUI (graphical user interface) that provides various options to help manage the process of transferring files.

To transfer files with FTP, you use a program often called the client. An FTP client program initiates a connection to a remote computer running FTP server software. After the connection is established, the client can choose to send and/or receive copies of files, singly or in groups. To connect to an FTP server, a client requires a username and password as set 
by the administrator of the server. Many public FTP archives follow a special convention for that accepts a username of "anonymous."

Simple FTP clients are included with most network operating systems, but most of these clients (such as FTP.EXE on Windows) support a relatively unfriendly command-line interface. Many alternative third-party FTP clients have been developed that support graphic user interfaces (GUIs) and additional convenience features. In any FTP interface, clients identify the FTP server either by its IP address (such as 192.168.0.1) or by its host name (such as ftp.about.com).

FTP supports two modes of data transfer: plain text (ASCII), and binary. You set the mode in the FTP client. A common error when using FTP is attempting to transfer a binary file (such as a program or music file) while in text mode, causing the transferred file to be unusable.

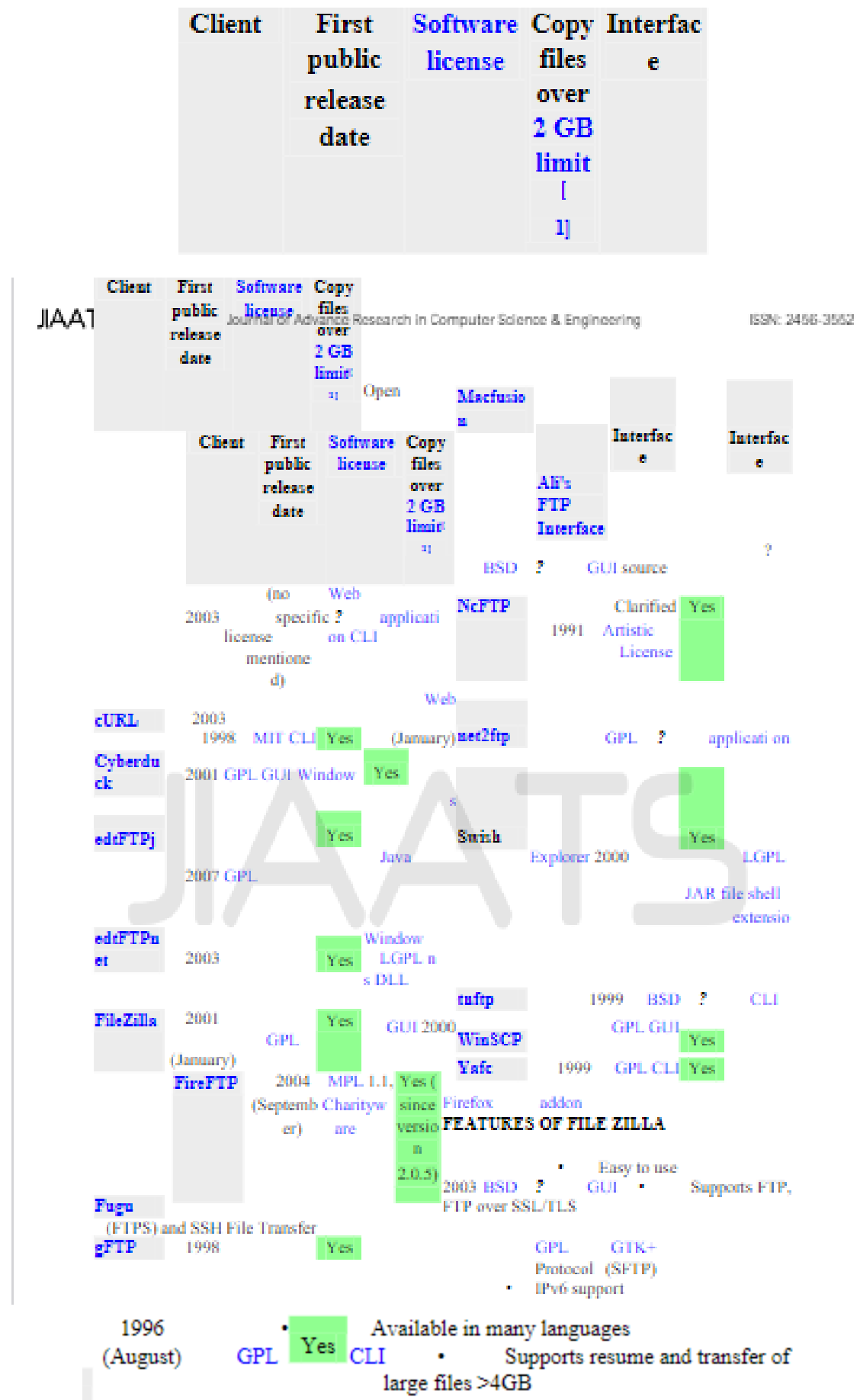

- Powerful Site Manager and transfer queue

- Bookmarks

- Configurable transfer speed limits

- Filename filters

- Directory comparison

- Network configuration wizard

- Remote file editing

- Keep-alive 
- Logging to file

- Remote file search

\section{TECHNOLOGY/PLATFORM OVERVIEW}

An edition of the Java platform is the name for a bundle of related programs from Sun which allow for developing and running programs written in the Java programming language. The platform is not specific to any one processor or operating system, but rather an execution engine (called a virtual machine) and a compiler with a set of libraries that are implemented for various hardware and operating systems so that Java programs can run identically on all of them.

- Java Card: A technology that allows small Java-based applications (applets) to be run securely on smart cards and similar smallmemoryfootprint devices.

- Java ME (Micro Edition): Specifies several different sets of libraries (known as profiles) for devices which are sufficiently limited that supplying the full set of Java libraries would take up unacceptably large amounts of storage.

- Java SE (Standard Edition): For general-purpose use on desktop PCs, servers and similar devices.

- Java EE (Enterprise Edition): Java SE plus various APIs useful for multitier client server enterprise applications.

\section{REFERENCE}

[1].Firewall 24/7 (Bpb)

[2].Data Communication (Forozon) (TMH)

[3].Networking Complete Reference (TMH)

[4].TCP/IP Protocol suite (Forozon) (TMH)

[5].Java Black book (Dreamtech)

[6].Network Programming with Java (O’rely)

[7].A Role-Based Trusted Network Provides Pervasive Security and Journal of Advance Research in Computer Science \& Engineering Compliance - interview with Jayshree Ullal, senior VP of Cisco

[8].Dave Dittrich, Network monitoring/Intrusion Detection Systems (IDS), University of Washington.

[9].Honeypots.net. 2007-05-26. Retrieved 2011-12-09.

[10]. "The six dumbest ways to secure a wireless LAN | ZDNet". Blogs.zdnet.com. Retrieved 201112-09.

[11]. Julian Fredin, Social software development program Wi-Tec 